\title{
ANALYSIS OF BOLT BENDING IN PRELOADED BOLTED JOINTS
}

\section{WELCH Michael ${ }^{1}$}

${ }^{1}$ Michael A Welch (Consulting Engineers) Limited, West Lancashire, UK, e-mail: mike.welch@mail.co.uk

\begin{abstract}
Classical analysis methods are applied to show how flexural deflections due to shear strain in the flange pack produce bending moments and tensile loads on bolts within preloaded bolted joints. It was found that in joints made with long bolts these loads can be significant. The loads can cause yielding of the bolt, reducing bolt preload. The methods presented are adequate to demonstrate the structural integrity of joints made with long bolts or with a small footprint.
\end{abstract}

KEYWORDS: bolted joint, preloaded bolt, bolt preload, bolt bending, long bolt

\section{Nomenclature}

$A_{b} \quad$ Tensile area of each bolt

$A_{j} \quad$ Total area of joint

$A_{\text {s.b }} \quad$ Shear area of each bolt

$d_{b} \quad$ Nominal bolt diameter

$d_{h} \quad$ Bolt hole diameter

$D_{b} \quad$ Effective diameter of bolt tensile area

$D_{n} \quad$ Basic effective diameter of bolt thread (pitch diameter)

$D_{s} \quad$ Minor diameter of bolt thread (root diameter)

$E_{b} \quad$ Young's Modulus of elasticity for bolt material

$F_{b_{(n)}} \quad$ Total axial bolt load on bolt ' $n$ '

$F_{p} \quad$ Preload in each bolt

$F_{s . b(n)} \quad$ Shear load on bolt ' $n$ '

$F_{\text {s.b.lim }(n)}$ Limiting shear load on bolt ' $n$ '

$F_{t . b(n)} \quad$ Tensile load on bolt ' $n$ '

$F_{x} \quad$ External In-plane force acting in $x$-direction

$F_{y} \quad$ External In-plane force acting in $y$-direction

$F_{z} \quad$ External axial load in direction of ' $z$ ' axis

$G_{f} \quad$ Shear Modulus for flange material

$I_{b} \quad$ Second Moment of Area of the tensile area of each bolt

$I_{b}^{\prime} \quad$ Second Moment of Area of the tensile area of a bolt

$I_{x x . j} \quad$ Second Moment of Area of joint about ' $x$ ' axis

$I_{x x . j}^{\prime} \quad$ Second Moment of Area transposed about $x$ '-axis

$I_{x y . j} \quad$ Product Moment of Area of joint 
$I_{y y . j} \quad$ Second Moment of Area of joint about ' $y$ ' axis

$J_{z z . j} \quad$ Polar Second Moment of Area of joint

$k_{j p} \quad$ Joint pack stiffness

$L_{f} \quad$ Through flange thickness

$L_{g} \quad$ Bolt grip length (including washers)

$M_{x} \quad$ External moment acting about the ' $x$ ' axis

$M_{x}^{\prime} \quad$ Resultant moment

$M_{y} \quad$ External moment acting about the ' $y$ ' axis

$M_{z} \quad$ External torsional moment acting on joint

$T_{p} \quad$ Residual torque in each bolt

$x_{(n)}, x_{s(n)}$ Coordinate of bolt ' $n$ '

$y_{(n)}, y_{s_{(n)}}$ Coordinate of bolt ' $n$ '

$y_{(n)}^{\prime} \quad$ Transposed coordinate of bolt ' $n$ '

$\propto \quad$ Flank angle of thread (half the included angle)

$\delta_{b(n)} \quad$ Displacement of bolt ' $n$ ' bolt head normal to bolt axis

$\delta_{z(n)} \quad$ Bolt Extension

$\theta \quad$ Angle of resultant moment

$\mu_{b} \quad$ Friction coefficient under bolt head

$\mu_{t} \quad$ Friction coefficient at thread flank

$\sigma_{a . b(n)} \quad$ Axial stress in bolt ' $n$ '

$\sigma_{b}(n) \quad$ Total tensile stress in bolt ' $n$ '

$\sigma_{b . b} \quad$ Bending stress component in each bolt

$\sigma_{m b}(n) \quad$ Total bending stress in bolt ' $n$ '

$\sigma_{m x} \quad$ Bending stress in bolt ' $n$ ' from moments about $x$-axis

$\sigma_{m y} \quad$ Bending stress in bolt ' $n$ ' from moments about $y$-axis

$\sigma_{s . b}(n) \quad$ Bending stress component in bolt ' $n$ '

$\sigma_{V M . c}(n) \quad$ Equivalent (Von Mises) stress at core of bolt ' $n$ '

$\sigma_{V M . r_{(n)}}$ Equivalent (Von Mises) stress at thread root of bolt ' $n$ '

$\tau_{b_{(n)}} \quad$ Shear stress in bolt ' $\mathrm{n}$ '

$\tau_{p} \quad$ Residual shear stress in each bolt

$\tau_{x(n)} \quad$ Shear stress at faying surface surrounding bolt ' $n$ ' from loads in $x$ direction

$\tau_{x y_{(n)}} \quad$ Shear stress at faying surface surrounding bolt ' $n$ '

$\tau_{y_{(n)}} \quad$ Shear stress at faying surface surrounding bolt ' $n$ ' from loads in $y$ direction 


\section{$1 \quad$ Introduction}

The detailed analysis of preloaded joints using classical theory of elasticity methods has been discussed in the paper "Classical Analysis of Preloaded Bolted Joint Load Distributions" [1]. This provided an understanding of how preloaded joints work and the interaction of the various components of the joint. Reference [1] considered the distribution of tensile loads on the bolts but did not go into depth on bolt bending.

Bolts with a low bending resistance, such as long bolts with a grip length several times greater than the nominal bolt diameter, can be prone to self loosening under transvers load reversals. Even with nut locking there can be a relaxation in bolt preload. Section 3.2.4 of VDI 2230 Part 1, "Systematic calculation of high duty bolted joints with one cylindrical bolt" [2] suggests that there could be up to $20 \%$ reduction in bolt preload.

The paper being presented here considers the classical analysis of bolt bending within preloaded bolted joints loaded with out-of-plane moments, in-plane loads and torsional moments. The effects these external loads have on bolt tensile stresses and their influence on bolt preload is discussed.

\section{Out-of-Plane Loads on the Joint}

Any external out-of-plane moments on the joint produces a stress gradient across the faying surface. The faying surface is the joints prepared contact face. This stress gradient produces a bending moment and bending stress component common to each of the bolts. The resulting bending stress component is given by:

$$
\sigma_{b . b}= \pm \frac{M_{x}}{I_{x x . j}} \cdot \frac{D_{b}}{2}
$$

It is common for the out-of-plane moment on a joint to be described by a pair of moments, $M_{x}$ and $M_{y}$, acting about the principle axes of the joint, or another convenient pair of perpendicular axes. These moments and their resultant, $M_{x}^{\prime}$, are illustrated in Figure (1).

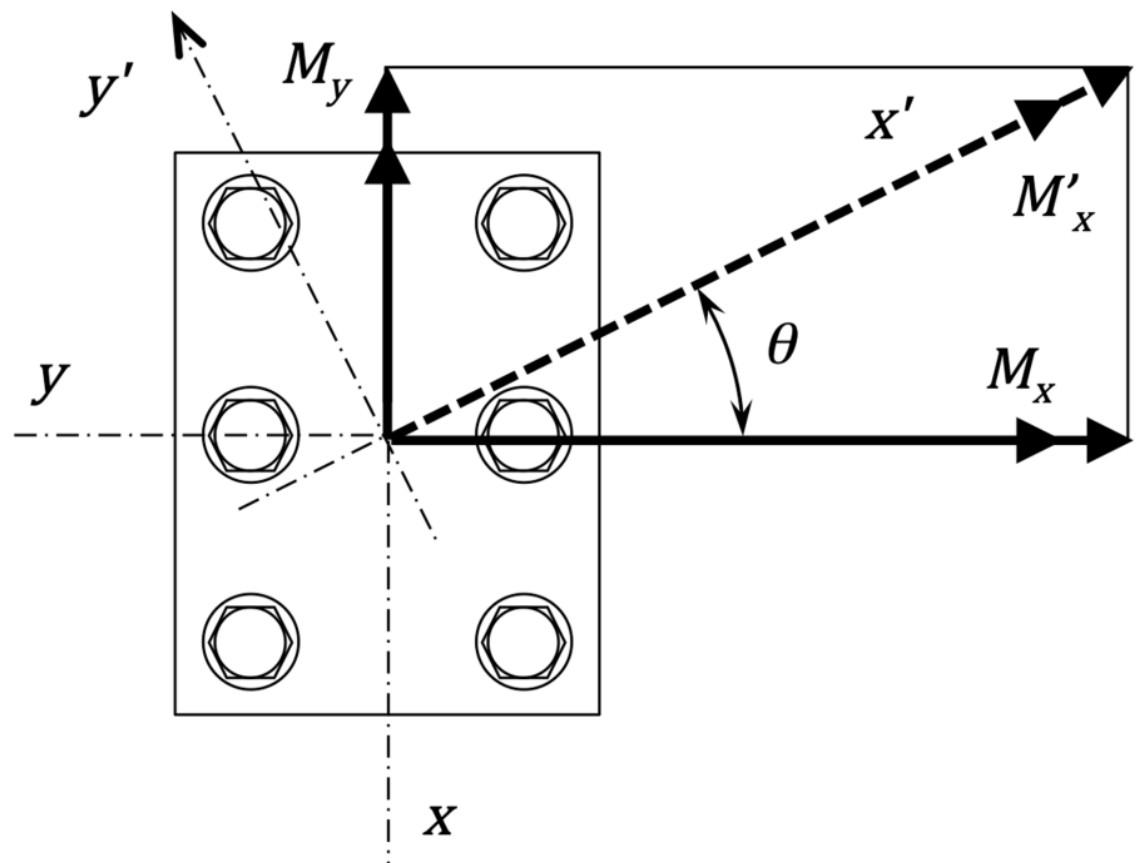

Fig. 1 Orientation of resultant moment. 
Since the resultant moment acts about a different axis to those used to define the joint, an alternative coordinate system, aligned to the resultant moment, needs to be considered. The angle between the transposed coordinate system and the joint coordinate system is given by:

$$
\theta=\arctan \left(M_{y} / M_{x}\right)
$$

If $M_{x}$ is negative then 180 degrees ( $\pi$ radians) needs to be added to the angle $\theta$ to ensure the direction of the resultant moment is in the correct 'quadrant'.

The resultant moment, $M_{x}^{\prime}$, is given by:

$$
M_{x}^{\prime}=M_{x} \cdot \cos (\theta)+M_{y} \cdot \sin (\theta)
$$

The Second Moment of Area about the $x$-axis transposed to the $x$ '-axis is given by:

$$
I_{x x . j}^{\prime}=I_{x x . j} \cdot \cos ^{2}(\theta)+I_{y y . j} \cdot \sin ^{2}(\theta)-I_{x y . j} \cdot \sin (2 \theta)
$$

Equations (2) to (4) follow the "right hand" rule, as illustrated in Figure (2). Loads are positive in the direction of the axes and positive moments act clockwise about the axes when viewed from the origin.

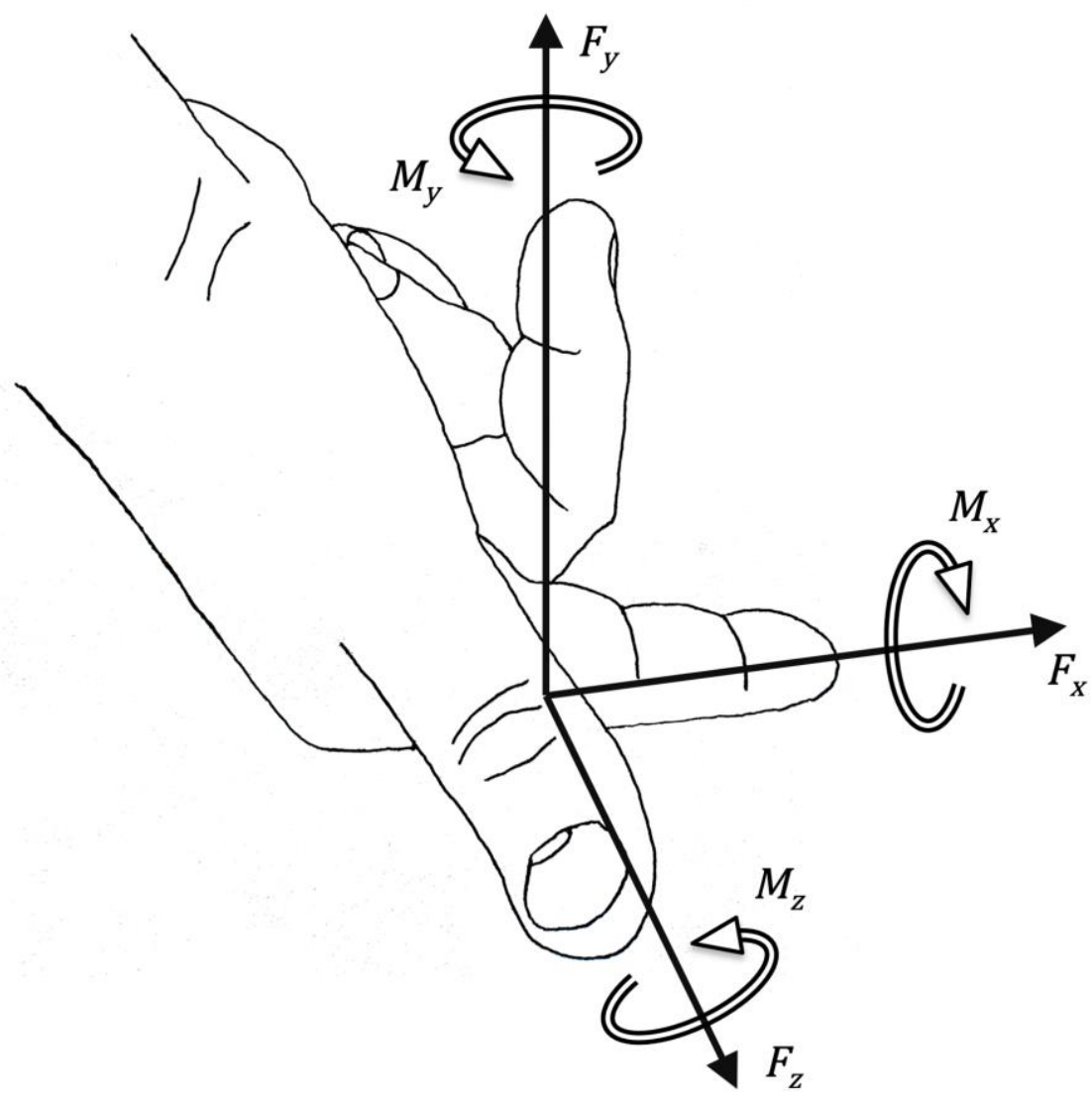

Fig. 2 Right hand rule coordinates.

When considering an out-of-plane moment that is not aligned with the joint $\mathrm{x}$-axis the terms for $M_{x}^{\prime}$ and $I_{x x . j}^{\prime}$ given by equations (3) and (4) should be used in equation (1). 
In most joints the bolt diameter is small compared to the overall size of the joint hence, the bending stress given by equation (1) is usually small compared to the bolt's axial stress. However, for joints with a narrow footprint, such as a single bolt or a single line of bolts, the bolt diameter may be almost the same as the width/length of the faying surface and this bolt bending stress can be significant.

\section{In-Plane Loads on the Joint}

External in-plane loads and torsional moments on the joint are supported by two mechanisms, friction at the faying surface and bolt shear. In some joints dowels, or other positive method of locating the joint, can assist these mechanisms.

Figure (3a) illustrates the way external in-plane loads are reacted into the bolts. The shear stresses in the flanges produced by the external in-plane loads and moment result in deflections at the bolt head, relative to the nut, perpendicular to the bolt axis. The shear loads producing these deflections are transmitted by friction under the bolt head/nut. The through thickness stiffness of the flanges is significantly greater than the flexural stiffness of the bolts. Hence, it is assumed that there is no flexural rotation of the bolt head or nut.

(a)

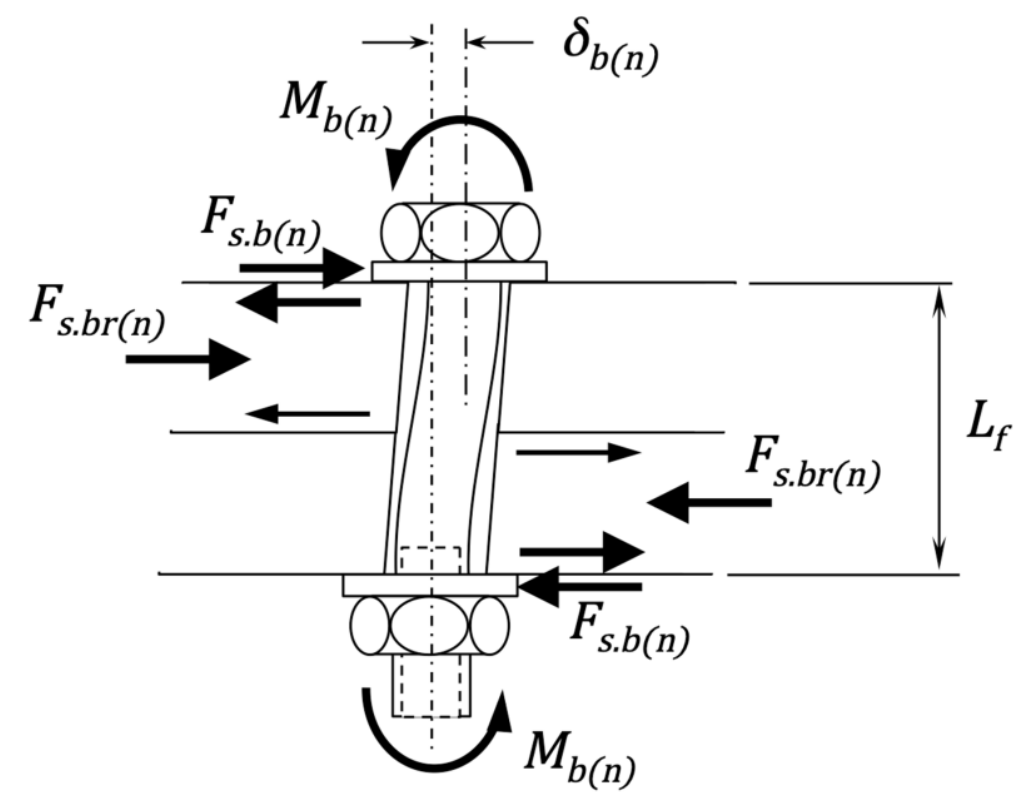

(b)

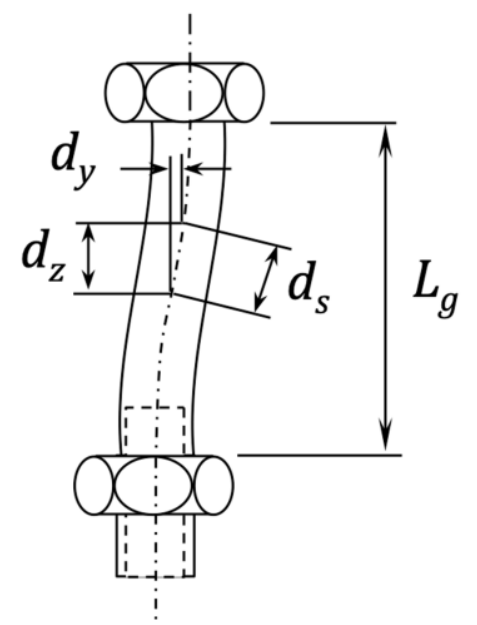

Fig. 3 Joint In-Plane loads reacted into a bolt.

The friction loads under the bolt head/nut, $F_{s . b_{(n)}}$, are normal to the axis of the bolt and produce bending moments on the bolt at the head and nut and a shear load on the shanks of each bolt within the joint. These shear loads exist even though the bolts do not bear on the sides of the holes in the flanges.

It was shown in reference [1] that, assuming there is no joint slip at the faying surface, the mean shear stress at the region of faying surface surrounding each bolt is given by the following three equations. 


$$
\begin{aligned}
& \tau_{x_{(n)}}=\frac{F_{x}}{A_{j}}-\frac{M_{z}}{J_{z z . j}} \cdot y_{s_{(n)}} \\
& \tau_{y_{(n)}}=\frac{F_{y}}{A_{j}}+\frac{M_{z}}{J_{z z . j}} \cdot x_{s_{(n)}} \\
& \tau_{x y_{(n)}}=\sqrt{\tau_{x_{(n)}^{2}}^{2}+\tau_{y_{(n)}^{2}}^{2}}
\end{aligned}
$$
joint.

Where $x_{s(n)}$ and $y_{s_{(n)}}$ are the coordinates of the bolt holes, relative to the centroid of the

Again, Equations (5) to (7) follow the "right hand" rule, as illustrated in Figure (2).

Equation (5) gives the shear stress component from loads acting in the $x$-direction. Similarly, equation (6) gives the shear stress component from loads acting in the $y$-direction. Equation (7) gives the resultant mean shear stress at the region/area of faying surface that surrounds the location of bolt ' $n$ '. This shear stress is carried across the faying surface by friction.

In an Ideal joint, preloading the joint's bolts induces a uniform compressive stress at the faying surface. In practice, the contact pressure will not be uniform across the surface. Each preloaded bolt influences an approximately circular region of the faying surface that surrounds it.

The bolt bending stresses calculated from equations (1) to (4) are influenced by the section properties $I_{x x . j}, I_{y y . j}$ and $I_{x y . j}$. These section properties can be calculated assuming the contact surface consists of the circular regions described above. However, the shear loads on the bolts calculated by equations (5) to (7) are determined by the shear strains in the joint pack. These shear strains are influenced by the whole of the joint pack. Hence, the section properties $A_{j}$ and $J_{z z . j}$ should be calculated from the geometry of the full flange section.

A method of calculating the area of the faying surface influenced by a bolt is presented in part 3, section 8.5 of Shigley's Mechanical Engineering Design [3] and in section 5.1.2 of VDI 2230 Part 1 [2].

Assume the transverse, normal to the bolt axis, flexural displacement of the bolt head arises from the shear strain across the thickness of the flanges. Then the displacement of bolt ' $\mathrm{n}$ ' is approximated by:

$$
\delta_{b_{(n)}}=\frac{\tau_{x y_{(n)}} \cdot L_{f}}{G_{f}}
$$

The bolt head displacement calculated by equation (8) is relative to the nut, not absolute.

The through flange thickness $L_{f}$ is the total flange thickness that carries the shear load. It does not include washers or packers under the bolt head or nut that do not directly react the external loads that produce in-plane shear in the flanges.

The bolt, as illustrated in figure (3a), is treated as a beam. Assuming the effective beam is fixed at the nut and there is no rotation at the bolt head then the shear load on the bolt, acting perpendicular to the bolt shank, is given by: 


$$
F_{s . b(n)}=\frac{12 \cdot E_{b} \cdot I_{b}}{L_{g}{ }^{3}} \cdot \frac{\tau_{x y_{(n)}} \cdot L_{f}}{G_{f}}
$$

The grip length $L_{g}$ is the total length of bolt between the contact faces of the bolt head and nut, including washers. It has been shown that the first internal thread supports a large proportion of the load and is subject to yielding [4]. Hence, an additional allowance of one bolt thread pitch can be added to the grip length to account for flexibility of the thread within the nut.

The Second Moment of Area for the bolt thread tensile section has been used in equation (9). This is the case for bolts threaded for their full length. When the bolts have two or more cross-sectional elements, such as a plain shank and threaded section then a mean effective Second Moment of Area could be used. The mean effective Second Moment of Area is given by:

$$
I_{b}^{\prime}=\frac{L_{g}}{\sum_{i} \frac{L_{(i)}}{I_{(i)}}}
$$

Where, $I_{(i)}$ and $L_{(i)}$ are the Second Moment of Area and Length respectively of each crosssectional element of the bolt.

The resulting shear stress in the bolt is given by:

$$
\tau_{b(n)}=\frac{F_{s . b(n)}}{A_{s . b}}
$$

The flexural stiffness of the bolts is considerably less than the torsional stiffness of the joint. Hence, shear stresses in the bolts are significantly less than the shear stress at the faying surface.

The bending stress at each bolt's thread root, at a location just under the nut, is given by:

$$
\sigma_{s . b(n)}= \pm \frac{F_{s . b(n)} \cdot L_{g}}{2 \cdot I_{b}} \cdot \frac{D_{b}}{2}
$$

\subsection{Bending Induced Bolt Tension}

Figure (3b) illustrates how the flexural deflection of the bolt and the high through flange stiffness results in some axial extension of the bolt. The extended length of an element is:

$$
d s=\sqrt{d z^{2}+d y^{2}}
$$

Which can be approximated as:

$$
d s=d z+\frac{1}{2} \cdot\left(\frac{d y}{d z}\right)^{2} d z
$$

The extended bolt length is given by: 


$$
\text { Extended length }=L+\delta_{z}=\int_{0}^{L} d s
$$

Hence:

$$
\delta_{z}=\frac{1}{2} \cdot \int_{0}^{L}\left(\frac{d y}{d z}\right)^{2} d z
$$

Solving this leads to:

$$
\delta_{z(n)}=\frac{F_{s . b(n)}^{2} \cdot L_{g}{ }^{5}}{240 \cdot\left(E_{b} \cdot I_{b}\right)^{2}}
$$

Where the transverse force, $F_{s . b_{(n)}}$, is the shear force given by equation (9).

This axial extension results in an axial load in addition to the bolt preload. This additional tensile bolt load component is given by:

$$
F_{t . b(n)}=\frac{F_{s . b(n)}^{2} \cdot L_{g}{ }^{4} \cdot A_{b}}{240 \cdot E_{b} \cdot I_{b}{ }^{2}}
$$

The additional tensile load given by equation (13a) is based on an infinitely stiff joint pack. Allowing for the stiffness of the joint pack the equation is rewritten as:

$$
F_{t . b(n)}=\frac{F_{s . b_{(n)}}^{2} \cdot L_{g}{ }^{4} \cdot A_{b}}{240 \cdot E_{b} \cdot I_{b}{ }^{2} \cdot\left(1+\frac{A_{b} \cdot E_{b}}{k_{j p} \cdot L_{g}}\right)}
$$

Where $k_{j p}$ is the through thickness stiffness of the joint pack under the bolt head.

The joint pack contained within the grip length of a bolt can be considered as consisting of a number of elements. A typical joint would consist of four elements, a washer under the bolt head, two flanges and a washer under the nut. A joint made with a tapped, or threaded, component would typically consist of two elements, a washer under the bolt head and a single flange. These two examples are of the most common joint configurations but other joints could include additional flanges, packers and spacers. The overall stiffness of the joint pack is found by combining the stiffness's of each element and can be estimated from:

$$
k_{j p}=\frac{1}{\sum_{i} \frac{t_{(i)}}{A_{(i)} E_{(i)}}}
$$

Where, $t_{(i)}, A_{(i)}$ and $E_{(i)}$ are the thickness, Area and Young's Modulus of Elasticity respectively of each element of the joint pack.

More refined methods of calculating the joint pack stiffness are suggested in both section 5.1.2 of reference [2] and part 3, section 8.5 of reference [3].

Neglecting the effects of the joint pack stiffness will results in a slightly conservative (high) tensile load component. 


\section{Combined Bending}

The bending stresses $\sigma_{b . b(n)}$ and $\sigma_{s . b(n)}$ are from moments acting about different axes. The vector addition of the two stress components is performed by the following equations:

$$
\begin{gathered}
\sigma_{m x_{(n)}}=\sigma_{b . b} \cdot \sin (\theta)-\sigma_{s . b(n)} \cdot \frac{\tau_{y_{(n)}}}{\tau_{x y_{(n)}}} \\
\sigma_{m y_{(n)}}=\sigma_{b . b} \cdot \cos (\theta)+\sigma_{s . b(n)} \cdot \frac{\tau_{x_{(n)}}}{\tau_{x y_{(n)}}} \\
\sigma_{m b(n)}=\sqrt{\sigma_{m x_{(n)}^{2}}^{2}+\sigma_{m y_{(n)}^{2}}^{2}}
\end{gathered}
$$

Equation (14) gives the bending stress component about the $x$-axis. Equation (15) gives the bending stress component about the $y$-axis. Equation (16) gives the resultant bending stress component in the bolt.

\subsection{Total Bolt Load}

Reference [1] shows that the bolt axial stresses resulting from the combined loading of the preload, external axial load and external out-of-plane moment are given by:

$$
\sigma_{a . b(n)}=\frac{F_{p}}{A_{b}}+\frac{F_{z}}{A_{j}}+\frac{M_{x}}{I_{x x . j}} \cdot y_{(n)}
$$

When considering an out-of-plane moment that is not aligned with the joint $\mathrm{x}$-axis the terms for $M_{x}$ and $I_{x x . j}$ in equation (17) should be replaced with $M_{x}^{\prime}$ and $I_{x x . j}^{\prime}$ as given by equations (3) and (4) and the term for $y_{(n)}$ should be replaced by:

$$
y_{(n)}^{\prime}=y_{(n)} \cdot \cos (\theta)-x_{(n)} \cdot \sin (\theta)
$$

Where $x_{(n)}$ and $y_{(n)}$ are the coordinates of bolt ' $n$ ' defined with respect to the centroid of the bolt group.

The total axial load on each bolt is given by:

$$
F_{b_{(n)}}=\sigma_{a . b(n)} \cdot A_{b}+F_{t . b(n)}
$$

The total bolt stress, including the bending stresses, is given by:

$$
\sigma_{b_{(n)}}=\frac{F_{b_{(n)}}}{A_{b}} \pm \sigma_{m b}(n)
$$

The localised increase in bolt stress, above the preload stress, may cause plastic deformation in the bolt thread that could result in some relaxation of the bolt preload.

\subsection{Bolt Limit of Proportionality}

The increase in bolt tension and bending stresses due to the external loads could produce some plastic deformation that could lead to relaxation of the bolt preload. The Von Mises yield criterion can be applied to both the core and thread root of the bolt. 
In addition to the stresses produced by the external loads each bolt will also have residual stresses from the axial preload and from some residual bolt tightening torque being locked in the bolt shank. Appendix B of BS 3580:1964 "Guide to the design considerations on: The strength of screw threads" [5] describes the bolt torque-tension relationships. From these relationships it is concluded that the residual torque in each bolt is:

$$
T_{p}=F_{p} \cdot \frac{D_{n}}{2} \cdot \frac{\mu_{t}}{\cos (\alpha)}
$$

The residual shear stress is given by:

$$
\tau_{p}=\frac{16 \cdot T_{p}}{\pi \cdot D_{s}^{3}}
$$

The Von Mises, or equivalent, stress in the bolt is given by the following two equations.

$$
\begin{gathered}
\sigma_{V M . c(n)}=\sqrt{\left(\frac{F_{b_{(n)}}}{A_{b}}\right)^{2}+3 \cdot\left(1.5 \cdot \tau_{b_{(n)}}+\tau_{p}\right)^{2}} \\
\sigma_{V M . r_{(n)}}=\sqrt{\sigma_{b_{(n)}}^{2}+3 \cdot\left(\tau_{b_{(n)}}^{2}+\tau_{p}^{2}\right)}
\end{gathered}
$$

Equation (23a) gives the Von Mises stress at the core of bolt ' $n$ '. The peak shear stress, at the centre of the bolt section, will be 1.5 times the mean shear stress.

Equation (23b) gives the Von Mises stress at the thread root. For this equation it has been assumed that some bolt shear stress will exist in the bolt thread root local to the nut, along with the residual shear stress.

\section{Joint Slippage}

In the previous sections it has been assumed that there is no joint slippage. This will be the case if dowels have been used to assist in carrying the in-plane loads by "pegging" the joint. However, if joint is not doweled then dynamic or impact loads may induce some joint slip.

Joint slippage is most likely to occur at the bolts where external out-of-plane loads and moments reduce the contact pressure at the faying surface. In doweled joints any slippage would take the form of localised slip as the shear stress at the faying surface is relaxed. In none doweled joints the slip could be more significant.

\subsection{Slip Limited by Displacement}

If the joint is not dowelled, the maximum flexural deflection of the bolt head relative to the nut is limited by the bolt and hole diameters. The bolt shear load due to transverse deflection of the bolt head is given by:

$$
F_{\text {s.b.lim }}=\frac{12 \cdot E_{b} \cdot I_{b}}{L_{g}{ }^{3}} \cdot\left(\frac{d_{h}-d_{b}}{2}\right)
$$

In deriving equation (24a) it is assumed that the bolt and hole axes are aligned prior to slip occurring. Under extreme geometric tolerance stack up of the joint assembly the bolt shear load, $F_{\text {S.b.lim }}$, could potentially be doubled. 
The limiting shear load, $F_{\text {s.b.lim(n) }}$, calculated by equation (24a) can be used in equations (10) and (11) in place of the shear load, $F_{S . b}(n)$, given by equation (9).

\subsection{Slip Limited by Friction}

In an extreme case slippage may occur between the bolt head or nut and the washer/flange. In this instance the load perpendicular to the bolt shank will be limited by friction under the bolt head or nut. It was shown in reference [1] that external loads and moments are supported mainly by a reduction in contact pressure at the faying surface, with only a small proportion of the external loads producing changes in the bolt tensile stresses. Hence, it is assumed that the limiting bolt shear load for an extreme case is given by:

$$
F_{\text {s.b.lim }}=\mu_{b} \cdot F_{p}
$$

Where $\mu_{b}$ is the friction coefficient under the bolt head or nut.

Soon after the installation of the bolt the friction coefficient $\mu_{b}$ will be that produced by any lubricant used during assembly. However, the value of the friction coefficient may change with the age, environment and history of the joint.

Again, the limiting shear load, $F_{\text {s.b.lim }}(n)$, calculated by (24b) can be used in equations (10) and (11) in place of the shear load, $F_{S . b}(n)$, given by equation (9).

\section{CONCLUSION}

External out-of-plane moments produce a stress gradient across the faying surface. This results in a bending stress component on each of the bolts, common to all of the joint's bolts. In joints with narrow footprints, such as a single bolt or a single line of bolts, the stress gradient and resulting bending stress can become significant.

The transvers flexural displacement of the bolt head produces a tensile load component in the bolt in addition to the existing preload. The total tensile load in the bolt may cause the limit of proportionality for the bolt material to be exceeded. This could lead to permanent set in the bolt, causing a relaxation of the bolt preload. This is a significant problem in joint assemblies incorporating long bolts.

The low flexural stiffness of a long bolt assembly means that, if the joint is not dowelled, the joint is more prone to slippage than one made using short bolts.

The bolt bending analysis discussed is suitable for calculating stresses for use in a fatigue analysis. In which case, stress concentration factors may also need to be applied to the calculated bolt stresses.

\section{REFERENCES}

[1] M.Welch. Classical Analysis of Preloaded Bolted Joint Load Distributions. International Journal of Structural Integrity 2018 (9), No. 4, 455 - 464.

[2] Verein Deutscher Ingenieure (Association of German Engineers). VDI 2230 Part 1. "Systematic calculation of high duty bolted joints with one cylindrical bolt", Verein Deutscher Ingenieure, Dusseldorf, 2003.

[3] R. Budynas, J. K. Nisbett. Shigley's Mechanical Engineering Design. $8^{\text {th }}$ edition. McGraw Hill, Primis Online. 2006, 2006, ISBN 0-390-76487-6. 
[4] M. Pástor, et al. The use of Optical Methods in the Analysis of Areas With Stress Concentration. Journal of Mechanical Engineering - Strojnicky časopis 2018 (68), No. $2,61-76$.

[5] British Standards Institution. BS 3580:1964, Guide to the design considerations on: The strength of screw threads, British Standards Institution, London, 1964. 